Original Research Article

\title{
A recent advance in first hour feeding - breast crawl
}

\author{
Tiwari $V^{1}$, Purohit $\mathrm{A}^{2}$, Verma $\mathbf{M}^{3}$ \\ ${ }^{1}$ Dr. Varsha Tiwari, Assistant Professor, Department of Pediatrics, ${ }^{2}$ Dr. Ashish Purohit, Assistant Professor, \\ Department of Medicine. Chattisgarh Institute of Medical Sciences, Bilaspur (CG), India, ${ }^{3}$ Mrs Mamta Verma, \\ Associate Professor, AIIMS College of Nursing, Bhopal, MP, India.
}

Address for Correspondence: Dr Varsha Tiwari, Email: dr.varshatiwari021@gmail.com

\begin{abstract}
Introduction: Ideally the baby should receive breast feed as soon as possible and preferably with in one hour of birth. Breast milk is right of every new born. Initiation of breast feeding in early hours, very minute after birth is crucial. Material and methods: A prospective cohort observation study done on 200 research cases \& similarly control cases were selected from full term newborn babies delivered vaginally, in one of the tertiary care teaching hospital of central India. Results: Out of 200 full term babies, 166 babies able to successfully crawl within in 50 min. temperature maintenance were found in $98 \%$ of $\mathrm{BC}$ babies and $64 \%$ of non $\mathrm{BC}$ babies. Blood sugar normal in $100 \%$ of $\mathrm{BC}$ babies and $35 \%$ of non $\mathrm{BC}$ babies. $100 \%$ of breast crawl babies were healthy and $95 \%$ of $\mathrm{BC}$ babies achieved good weight gain after 15 days follow up. Conclusions: Breast crawl helps in faster effective achievement of feeding skill by the baby, getting colostrums, which has many antibodies and antiinfective properties, prevent hypothermia as well as hypoglycemia and boosting neurodevelopment of babies nervous system.
\end{abstract}

Keywords: Breast crawl, Breast feeding, Hypoglycemia, Hypothermia

\section{Introduction}

Mother-infant separation postbirth is common in Western culture. Early skin-to-skin contact (SSC) begins ideally at birth and involves placing the naked baby, head covered with a dry cap and a warm blanket across the back, prone on the mother's bare chest. According to mammalian neuroscience, the intimate contact inherent in this place (habitat) evokes neurobehaviors ensuring fulfilment of basic biological needs. This time may represent a psychophysiological 'sensitive period' for programming future physiology and behaviour [1]

Every new born, when placed on her mother's abdomen, soon after birth, has the ability to find her mother's breast all on her own and to decide when to take the first breastfeed. This is called the 'Breast Crawl'. It was first described in 1987 at the Karolinska Institute in Sweden (Windstorm et al, 1987) [2]. Breastmilk makes the world healthier, smarter, and more equal: these are the conclusions

Manuscript received: $8^{\text {th }}$ August 2017

Reviewed: $18^{\text {th }}$ August 2017

Author Corrected: $26^{\text {th }}$ August 2017

Accepted for Publication: $30^{\text {th }}$ August 2017 of a new Lancet Series on breastfeeding. The deaths of 823000 children and 20000 mothers each year could be averted throughuniversal breastfeeding, along with economic savings of US\$300 billion[3].

Breastfeeding obviously needs no further justification,but the ways of getting it to work remain very much understudy; we need to know what's going to be the most effective way in helping to offset the long trend away frombreastfeeding. Regarding micronutrient supplements, weneed to learn how to make sure that children are gettingvitamin A and iodine and other interventions that we knowwork, but where we have yet to crack the nut of how to getthem to scale [4].

The human female's nipple-areolar complex (NAC) is the point of arrival of a natural progression from birth to breastfeeding, linked to functional, chemical and biophysical cues that promote the breastcrawl soon after birth [5]. For the first time, that a temperature gradient may support mother-infant thermal identification and communication in the 
breastcrawl and in the natural progression of the continuum from birth to breastfeeding [5].

Mother-infant separation post birth is common. In standard hospital care, newborn infants are held wrapped or dressed in their mother's arms, placed in open cribs or under radiant warmers. Skin-to-skin contact (SSC) begins ideally at birth and should last continually until the end of the first breastfeeding. SSC involves placing the dried, naked baby prone on the mother's bare chest, often covered with a warm blanket.

According to mammalian neuroscience, the intimate contact inherent in this place (habitat) evokes neurobehaviors ensuring fulfilment of basic biological needs. This time frame immediately post birth may represent a 'sensitive period' for programming future physiology and behaviour[1].

So we're very hopeful that the moment is here, and nowit's up to us to capitalize on this to make a lasting differencefor everybody, starting with this most powerful interventionof all, breastfeeding.

\section{Material and Methods}

Settings- Department of paediatrics\& neonatology and Department of obstetrics and gynaecology in one of the tertiary care teaching hospital of central India.

Study Design- A prospective cohort observational study on 200 babies born in Dr BRAM Hospital Raipur CG.

Inclusion criteria- 200 newborn babies of gestational age more than 36 weeks, with Apgar score above 6, weight above 1800 gm, normal and healthy, born by normal vaginal deliveries in labour room of dept of obstetrics and gynaecology medical college Raipur were selected.

Exclusion criteria- Those babies born of out born and inborn LSCS, Apgar $<6$, weight $<1800$ gm and with congenital anomalies were excluded.

Summary of The Procedure- Procedure for Breast Crawl for optimum results

Discuss it with the mother during antenatal visits and Orient the staff to the technique of breast crawl. Avoid labour analgesia as far as possible. After delivery if the baby has cried well immediately after birth and is stable she/he does not need oro-nasal suction. Dry the baby nicely except for the hands. Raise the mother's head on the pillow so that the mother can see her baby easily during the Breast Crawl.Show the baby to the mother with a cheek to cheek contact. The mother may like to whisper a holy message to the baby. Now keep the naked baby on the mothers abdomen with the head in between the bare, unwashed and unwiped breast. Cover the baby and mother together with a cloth, to keep warm, while skin to skin contact continues. The mothers hand can support the baby's back.

This will help in maintaining the baby's position, avoid slipping of the baby and give the added advantage of maternal touch.Continue in this position till the baby takes the first breast feed; which most will achieve by 30-60 minutes.In case the baby's several attempts to latch on the breast fail, then the baby can be gently moved nearer to the breast and assisted by a helper to attach to the breast. The mother should not be moved out of the labour room till the first breast feed is completed.A female relative or even the father can be called in at the earliest and acceptable time to be a part of this important magical emotional interaction.

Breast odour is strong stimulus which drives the baby towards nipple. The babies sense of smell is well developed. The odour of a substance secreted by nipple is similar to the smell of a substance in amniotic fluid which surrounds the baby womb. Nipple massage by the baby makes it protract. This helps in attachment. Nipple massage also releases hormone called oxytocin in mother, this helps to contract the uterus, prevent the bleeding and reduce the maternal anaemia. baby start to make mouthing movement.

Babies hand should have amniotic fluid on them, as it guides baby to nipple. babies shoulder, hip and neck muscles are sufficiently developed to help the baby to move. Even with its limited vision baby can see areola. If baby raises its head it can also see her mothers face also. The baby then reaches nipple, raises her head and gets nicely attached on to the nipple with her mouth widely open to take a mouthful of breast. This first skin to skin contact must continue until the baby finishes the first breast feed. This is the first feed to the babies that gives moment of joy for mother and baby over a ten 
minutes and create higher human potential of successful growth and development, cuddle, love, security and mother baby bonding.

Instruments used- babies were resuscitated using standard protocol, babies weighed using standard electronic weighing machine, blood sugar measured with the help of glucose strips by heel prick method using bets check glucometer.

Statistical analysis- Odds ratio and $\log$ odds ratio with chi square test of significance were used for statistical analysis of data statistical test of significance was defined as $\mathrm{p}<0.05$.

\section{Results}

It was observed that out of 200 babies, 166 were able to reach the breast of mother by crawling with in $30-50$ minutes of time.

Table No.-1: Effect of temperature on breast crawl.

\begin{tabular}{|c|c|c|c|c|}
\hline & \multicolumn{2}{|c|}{ Maintain temperature (36.37.c) } & \multicolumn{2}{c|}{ Not maintain temperature(35-36.c) } \\
\hline & No. & $\%$ & No. & $\%$ \\
\hline Breast crawl & 163 & 98 & 3 & 2 \\
\hline Non breast crawl & 22 & 64 & 12 & 36 \\
\hline
\end{tabular}

The temperature maintenance were found in $98 \%$ of $\mathrm{BC}$ babies and $64 \%$ of non $\mathrm{BC}$ babies, where as temperature were not maintained in $2 \%$ of breast crawl babies and $36 \%$ of non breast crawl babies.

Table No.-2: Effect of blood sugar on breast crawl.

\begin{tabular}{|c|c|c|c|c|}
\hline & \multicolumn{2}{|c|}{ Normoglycemia (>40 mg/dl) } & \multicolumn{2}{c|}{ Hypoglycaemia (<40 mg/dl) } \\
\hline & No. & $\%$ & No. & $\%$ \\
\hline Breast crawl & 166 & 100 & 0 & 0 \\
\hline Non breast crawl & 12 & 35 & 22 & 75 \\
\hline
\end{tabular}

Blood sugar normal in 100\% BC babies and 35\% non $\mathrm{BC}$ babies whereas hypoglycaemia was found in $75 \%$ non BC babies.

Table No.-3: Effect of crying episode on breast crawl

\begin{tabular}{|c|c|c|c|c|}
\hline & \multicolumn{2}{|c|}{ Less crying episode (<2min) } & \multicolumn{2}{c|}{ More crying episode(>2 min) } \\
\hline & No. & $\%$ & No. & $\%$ \\
\hline Breast crawl & 158 & 95 & 8 & 5 \\
\hline Non breast crawl & 6 & 17 & 28 & 83 \\
\hline
\end{tabular}

It has been found that there is decreased duration of crying episode i.e $<2$ min in $95 \%$ of $\mathrm{BC}$ babies and $17 \%$ of non $\mathrm{BC}$ babies and more crying was found in $5 \%$ of $\mathrm{BC}$ and $83 \%$ of non $\mathrm{BC}$.

Table No-4: Effect of sickness of baby on breast crawl.

\begin{tabular}{|c|c|c|c|c|}
\hline & \multicolumn{2}{|c|}{ Healthy baby } & \multicolumn{2}{c|}{ Sick baby } \\
\hline & No. & $\%$ & No. & 0 \\
\hline Breast crawl & 166 & 100 & 0 & 12.7 \\
\hline Non breast crawl & 30 & 88 & 4 & 0 \\
\hline
\end{tabular}

It has been found that $100 \% \mathrm{BC}$ babies are healthy while $88 \%$ of non $\mathrm{BC}$ are healthy, none of $\mathrm{BC}$ are healthy, none of $\mathrm{BC}$ babies were sick. 
Original Research Article

Table No.-5: Study of weight gain in BC babies after 15 days follow up.

\begin{tabular}{|c|c|c|c|c|}
\hline & \multicolumn{2}{|c|}{ Weight gain } & \multicolumn{2}{c|}{ Non weight gain } \\
\hline & No. & $\%$ & No. & 5 \\
\hline Breast crawl & 158 & 95 & 8 & 71 \\
\hline Non breast crawl & 10 & 29 & 24 & 5 \\
\hline
\end{tabular}

It has been found that $95 \%$ of $\mathrm{BC}$ babies achieved good weight gain after 15 days follow up.

\section{Discussion}

Initiation of breast feeding within hour of birth is very crucial. As soon as baby born in mammalian family nature is provided for their immediate feeding of newborn.Except human other mammalian species start immediate breast feeding of their newborn. Breast milk is the right of every newborn. Initiation of breast feeding in early hours, very minute after birth is crucial.

Aghdas K et al [6] conducted randomised control study toevaluate the effect of mother-infant immediate skin-to-skin contact on primiparous mother's breastfeeding self-efficacy. They did Skinto-skin contact immediately after birth and then controlling breastfeeding self-efficacy at 28 days postpartum, concluded that Mother-infant immediate skin-to-skin contact is an easy and available method of enhancing maternal breastfeeding self-efficacy. High breastfeeding selfefficacy increases exclusive breastfeeding duration, this supports our study.

Ruth cantriletal et al [7] found that sustained naked body contact with attention to newborn instinctive feeding behaviours may improve breastfeeding outcomes for women \& boost their breastfeeding confidence at 2 wks postpartum.

Kristin E Svensson[8] found that Skin-to-skin contact during breastfeeding seems to immediately enhance maternal positive feelings and shorten the time it takes to resolve severe latch-on problems in the infants who started to latch.

An underlying mechanism may be that skin-to-skin contact with the mother during breastfeeding may calm infants with earlier strong reaction to "hands on latch intervention" and relieve the stress which may have blocked the infant's inborn biological program to find the breast and latch on. CondeAgudeloA et al [9] found that Evidence from this updated review supports the use of KMC in LBW infants as an alternative to conventional neonatal care, mainly in resource-limited settings. Further information is required concerning the effectiveness and safety of early-onset continuous KMC in unstabilized or relatively stabilized LBW infants, as well as long-term neurodevelopmental outcomes and costs of care.

Matthiesen AS et al [10] studied on Ten vaginally delivered infants whose mothers had not been exposed to maternal analgesia were video-recorded from birth until the first breastfeeding. Video protocols were developed based on observations of the videotapes. Each infant's hand, finger, mouth, and tongue movements, positions of the hand and body, and sucking behaviour were assessed every 30 seconds. Maternal blood samples were collected every 15 minutes, and oxytocin levels were analysed by radioimmunoassay.

A statistical test for establishing the relationship between maternal oxytocin levels and infants' hand movements or sucking behaviour was developed. Infants used their hands to explore and stimulate their mother's breast in preparation for the first breastfeeding. A coordinated pattern of infant hand and sucking movements was also identified. When the infants were sucking, the massagelike hand movements stopped and started again when the infants made a sucking pause. Periods of increased massagelike hand movements or sucking of the mother's breast were followed by an increase in maternal oxytocin levels $(\mathrm{p}<0.005)$.

The findings indicate that the newborns use their hands as well as their mouths to stimulate maternal oxytocin release after birth, which may have significance for uterine contraction, milk ejection, and mother-infant interaction[10]. This supports crawling of baby over mother's abdomen in our study. 
Nemsadze $\mathrm{K}$ et al [11] found that Attachment between mother and child is the unique and complex type of relationship that ensures safety and nurturing of a child in the beginning and effective functioning and ability to establish relationships later on. Maternal behaviour is generally known to be influenced by multiple psychosocial factors. Series of the studies performed in animal models demonstrated that decline in progesterone level on the background of increasing estradiol concentration prepares brain for the action of progesterone and prolactin that are responsible for the rapid onset of maternal behaviour postpartum.

However the results of another group of researchers failed to prove the unique importance of oxytocin. Other substances such as epinephrine, norepinephrine, serotonin were also suggested though diversity in the study results interferes with making definitive conclusions. This facilitates neuroendocrine relation with breast crawl in our study.

The revised standard of care for breastfeeding infants at risk of developing hypoglycemia during transitioning to extrauterine life was developed using the American Academy of Pediatrics (AAP) 2011 hypoglycemia guidelines, the Academy of Breastfeeding Medicine protocol, and staff input. A pre/postimplementation chart audit indicated support of infant safety by glucose stabilization, breastfeeding within the first hour of life, and breastfeeding frequency without an increase in blood sampling, formula use, or admissions to the special care nursery $[12,13]$. The concept of "Golden 60 minutes" or "Golden Hour" has been derived from adult trauma. It has been defined as the first $60 \mathrm{~min}$ of postnatal life. It has been seen that care received by any newborn in the initial first hour has implications in the future life, showing the importance of golden hour [14]. In golden hour approach for term newborn, the importance is given to effective and evidence based resuscitation, postresuscitation care, delayed cord clamping, prevention of hypothermia, immediate breast feeding, prevention of hypoglycemia, and starting of therapeutic hypothermia in case of moderate to severe asphyxia. In this part of review, we will cover all the golden hour interventions in term neonate with current evidence [14]. This golden hour is also valuable for breast crawl as we did in our study.
Srivastava $\mathrm{S}$ et al[15] observed that SSC contributed to better suckling competence as measured by IBFAT score $(\mathrm{P}<0.0001)$. More babies in the SSC group were exclusively breastfed at first follow-up visit $(\mathrm{P}=0.002)$ and at 6 weeks $(\mathrm{P}<0.0001)$. SSC led to higher maternal satisfaction rates, better temperature gain in immediate post-partum period, lesser weight loss was at discharge and at first follow-up (all $\mathrm{P}<0.0001$ ) and lesser morbidity than the study group $(\mathrm{P}=0.006)$. Very early SSC is an effective intervention that improves baby's suckling competence, maternal satisfaction, breastfeeding rates and temperature control and weight patterns, Which is similar to our study.

Mahmood I et al [16] studied onEligible mothers, were assessed for the successful breastfeeding by using IBFAT tool. The time to initiate the first feed, time to effective breastfeeding, maternal satisfaction with the care provided, preference for the same care in future and level of exclusive breastfeeding at the age of one month were also noted. The data was compared by using X2 and t-test. Significant p-value was taken as $<0.05$. Maternal-infant early skin-toskin contact significantly enhanced the success of first breastfeed and continuation of exclusive breastfeeding till one month of age. It also reduced the time to initiate first feed and time to effective breastfeeding. cochrane review found that found low-quality evidence that healthcare professionalled breastfeeding education and non-healthcare professional-led counselling and peer support interventions can result in some improvements in the number of women beginning to breastfeed.

These might include well-described interventions, including health education, early and continuing mother-infant contact, and initiatives to help mothers overcome societal barriers to breastfeeding, all with clearly defined outcome measures [17].

FeferbaumR[18] showed that health education and peer support interventions can result in some improvements in the number of women beginning to breastfeed. Findings from these studies suggest that larger increases are likely to result from needsbased, informal repeat education sessions than more generic, formal antenatal sessions. These findings are based only on studies conducted in the USA, among women on low incomes with varied ethnicity and feeding intention, and this raises some questions regarding generalizability to other settings. 
Ginovart $\mathrm{G}$ et al[19]studied on newbornand found that Outcomes revealed that an initial human milk diet with standard fortification was associated with significantly higher early extrauterineweight gain and head growth in very low-birth-weight infants than a formula-fed diet. This supports our study of weight gain in newborn of breast crawl infants.

Klaus and Kennel et al [20] reviewed many of these studies and gave a beautiful description of breast crawl. Credit of using word breast crawl as 'noun' should be given to Klaus.

Breast crawl is associated with variety of sensory, central, motor and neuroendocrine component all directly or indirectly helping the baby to move and facilitate her survival in new work. Babies use taste and smell of amniotic fluid on its hand to make a connection with a lipid substance on nipple related to amniotic fluid.

Evidence supports the use of SSC to promote breastfeeding. Studies with larger sample sizes are necessary to confirm physiological benefit for infants during transition to extra-uterine life and to establish possible dose-response effects and optimal initiation time. Methodological quality of trials remains problematic, and small trials reporting different outcomes with different scales and limited data limit our confidence in the benefits of SSC for infants.

\section{Conclusion}

Breast crawl helps to reduce neonatal hypothermia, hypoglycemia as well as helps in faster achievement of feeding skill, getting colostrums, thus promotesweight gain and boosting neurodevelopment of baby.

Birth and immediate postpartum period pose many challenges for the newborn. The neonatal mortality rates are high in India, whereas the breastfeeding rates are still low. Hence, need exists for a simple and easily applicable intervention, which may counter these challenges.

Our review included only healthy infants, which limits the range of physiological parameters observed and makes their interpretation difficult.

Funding: Nil, Conflict of interest: None initiated, Perission from IRB: Yes

\section{Bibliography}

1. Widström AM, Lilja G, Aaltomaa-Michalias P, Dahllöf A, Lintula M, Nissen E. Newborn behaviour to locate the breast when skin-to-skin: a possible method for enabling early self-regulation. Acta Paediatr. 2011 Jan;100 (1):79-85. doi: 10.1111/j. 1651 - 2227. 2010.01983.x. Epub 2010 Sep 14.

2. Breastfeeding: achieving the new normal. Breastfeeding series: The Lancet: January 30, 2016. http:// www.thelancet.com

3. Hansen, K. The power of nutrition and the power of breastfeeding. Breastfeeding Med. 2015; 10: 385388. available on www.ncbi.nlm.nih.gov

4. Zanardo $\mathrm{V}^{1}$, Volpe $\mathrm{F}$ A temperature gradient may support mother-infant thermal identification and communication in the breast crawl from birth to breastfeeding. ActaPaediatr. 2017 Jul 19. doi: 10. 1111 / apa.13976.

5. Moore ER, Bergman N, Anderson GC, Medley N. Early skin-to-skin contact for mothers and their healthy newborn infants. Cochrane Database Syst Rev. 2016 Nov 25;11:CD003519.

6. Aghdas $\mathrm{K}^{1}$, Talat $\mathrm{K}$ Effect of immediate and continuous mother-infant skin-to-skin contact on breastfeeding self-efficacy of primiparous women: a randomised control trial. Women Birth. 2014 Mar; 27 (1):37-40

7. Ruth M Cantrill, Debra K Creedy. Effective suckling in relation to naked maternal-infant body contact in the first hour of life: an observation study. BMC Pregnancy Childbirth. 2014; 14: 20. clok. uclan.ac.uk

8. Kristin E Svensson,Marianne I Velandia.Effects of mother-infant skin-to-skin contact on severe latch-on problems in older infants: a randomized trial Int Breastfeed J. 2013; 8: 1.

9.Conde-AgudeloA ${ }^{1}$, Díaz-Rossello JL.Kangaroo mother care to reduce morbidity and mortality in low birthweight infants. Cochrane Database Syst Rev. 2016 Aug 23;(8).

10. Matthiesen AS, Ransjö-Arvidson AB. Postpartum maternal oxytocin release by newborns: 


\section{Original Research Article}

effects of infant hand massage and sucking. Birth. 2001 Mar; 28 (1):13-9.

11. Nemsadze K, Silagava M. Neuroendocrine foundation of maternal-child attachment. Georgian Med News. 2010 Dec;(189):21-6.

12. Csont GL, Groth S, Hopkins P, Guillet R. An evidence-based approach to breastfeeding neonates at risk for hypoglycemia. J ObstetGynecol Neonatal Nurs. 2014 Jan-Feb; 43 (1):71-81. doi: 10.1111/ 1552- 6909. 12272.

13. Bragg JJ, Green R Does early enteral feeding prevent hypoglycemia in small for gestational age neonates? J Neonatal Perinatal Med. 2013; 6 (2): 131-5.

14. Sharma D, Sharma P. Golden 60 minutes of newborn's life: Part 2: Term neonate. J MaternFetal Neonatal Med. 2016 Nov 29:1-6.

15. Srivastava S, Gupta A, Bhatnagar A, Dutta S. Effect of very early skin to skin contact on success at breastfeeding and preventing early hypothermia in neonates. Indian J Public Health. 2014 Jan-Mar; 58 (1) : 22-6. doi: 10.4103/0019-557X.128160.
16. Mahmood I, Jamal M, Khan N. Effect of mother-infant early skin-to-skin contact on breastfeeding status: a randomized controlled trial. J Coll Physicians Surg Pak. 2011 Oct; 21(10):601-5. doi: 10.2011/JCPSP.601605.

17. Balogun OO, O'Sullivan EJ, McFadden A, Ota E, Gavine A, Garner CD, Renfrew MJ, Mac Gillivray S.Interventions for promoting the initiation of breastfeeding. Cochrane Database Syst Rev. 2016 Nov 9;11:CD001688.

18. Feferbaum R.Interventions for promoting the initiation of breastfeeding. Sao Paulo Med J. 2014;132(1):68.

19. Ginovart G, GichI A Fortified Donor Milk Policy is Associated With Improved In-Hospital Head Growth and Weight Gain in Very Low-BirthWeight Infants. Adv Neonatal Care. 2017 Aug;17 (4): 250-257.

20. Klaus MH, Kennell JH, Plumb N, ZuehlkeS.Pediatrics. Human maternal behavior at the first contact with her young. 2001. breastcrawl.org

How to cite this article?

Tiwari V, Purohit A, Verma M. A recent advance in first hour feeding- breast crawl. J PediatrRes. 2017;4(08):537-543.doi:10. 17511/ijpr.2017.i08.07. 